\title{
A NOTE ON THE PROBLEM OF FINDING A $(3,9)$-CAGE
}

\author{
P. K. WONG \\ Department of Mathematics \\ Seton Hall University \\ South Orange, New Jersey 07079 U.S.A. \\ (Received April 21, 1985)
}

\begin{abstract}
In this paper, we discuss the problem of finding a $(3,9)$-cage. A hamiltonian graph with girth 9 and 54 vertices is given. Except four vertices, each of the remaining vertices of this graph has valency three. This graph is obtained with the aid of a computer.
\end{abstract}

KEY WORDS AND PHRASES. Cage, regular graph, hamiltonian graph. 1980 MATHEMATICS SUBJECT CLASSIFICATION CODE. 05C35

1. INTRODUCTION.

The problem of finding a smallest graph of girth 9 and valency $3(a(3,9)$ cage) is an old and difficult one. In 1952, R. M. Foster constructed the first trivalent graph with girth 9 and 60 vertices. Since then, at least 30 graphs of the same type have been obtained by various mathematicians. By using a computer, Biggs and Hoare [1] obtained a trivalent graph with girth 9 and 58 vertices. It is not known yet whether this graph is a $(3,9)$-cage.

Recently, with the aid of a computer, the author has completed the study of the $(3,10)$-cages [4], and he hopes to find a $(3,9)$-cage in this way. After considerable computation time was used in a computer, the author has obtained many partial solutions which are close to a $(3,9)$-cage. As an example, a hamiltonian graph with girth 9 and 54 vertices is given in this paper. Except four vertices, each of the remaining vertices of this graph has valency three. It is hoped that this graph may be useful in the search of a $(3,9)$-cage. 
2. A HAMILTONIAN GRAPH OF ORDER 54 .

Let $f(3,9)$ be the number of vertices of a $(3,9)$-cage. It is known that $54 \leq f(3,9) \leq 58$ (see [1]). The determination of the exact value of $f(3,9)$ is of interest. By using a method similar to that in [4], the author hopes to construct a $(3,9)$-cage. He came close to obtaining one on many occasions. As an example, a hamiltonian graph with girth 9 and 54 veritces is given here.

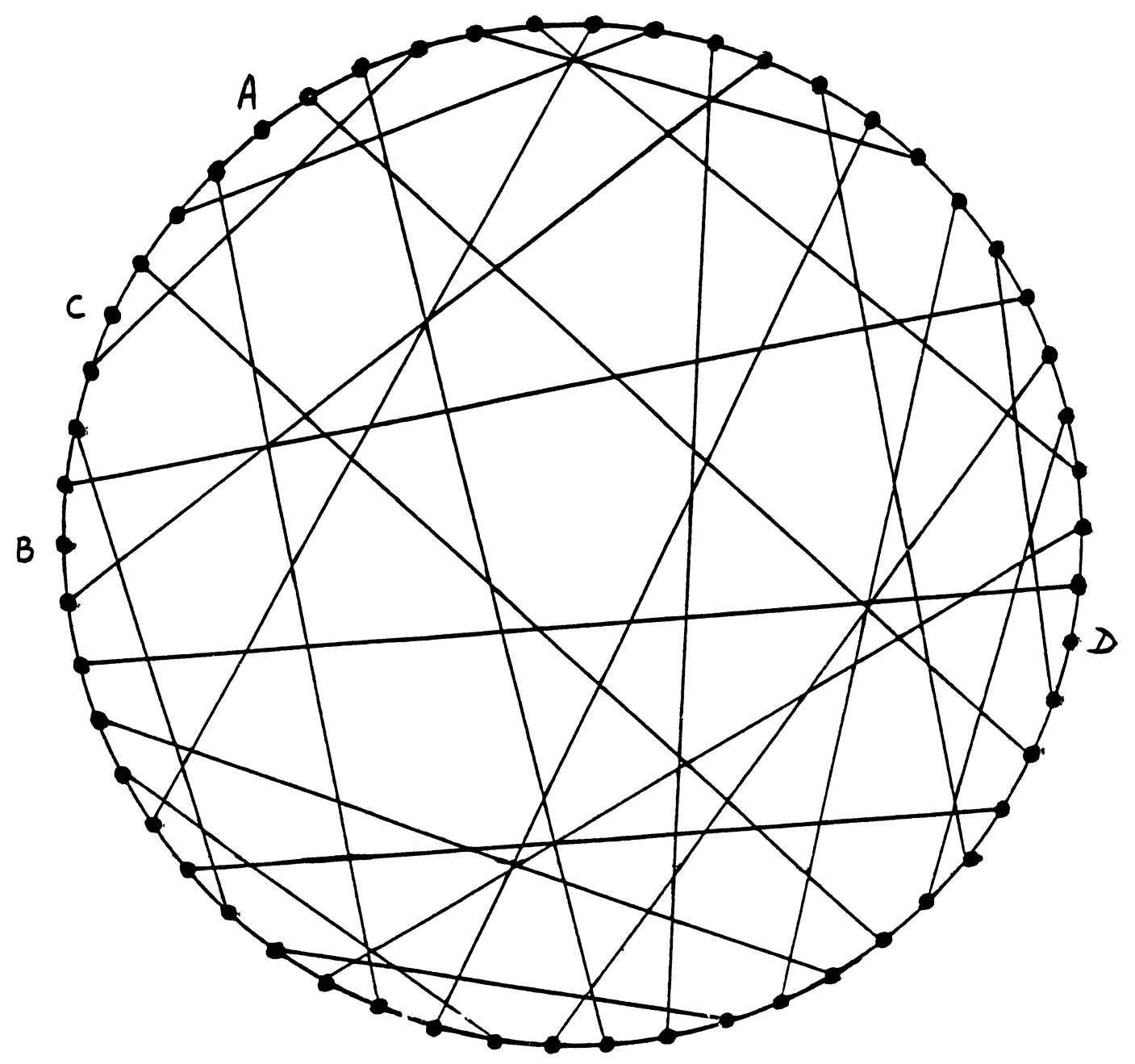

In the above graph, except vertices $A, B, C$ and $D$, each of the remaining vertices of the graph has valency three. If $A$ is adjacent to $B$ and $C$ is adjacent to $D$, then this graph becomes a trivalent graph of girth 8 .

In [1], Biggs and Hoare obtained a trivalent graph with girth 9 and 58 
vertices. The author has tried to obtain a symmetrical design of this graph by using the mathod suggested in [3]. For each vertex $v$ of this graph, we draw a tree $T_{v}$ of order 46 in which all internal vertices have valency 3 , containing the vertex $v$ in the middle. There are 12 vertices of the graph, which do not belong to $T_{v}$. These 12 vertices are called the opposite vertices (with respect to vertex $v$ ) (see [2] and [3]). These 12 opposite vertices form 17 different cases of arrangement, which are given in the figure below. (A simple computer program can be used to obtain this information.)

(1)..
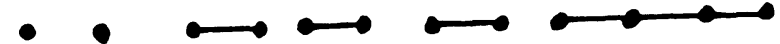

1,36

(2).

(3).
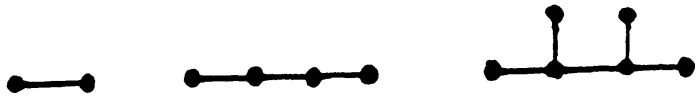

$2,23,35,58$
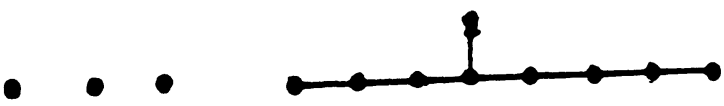

$3,22,51,57$

(5).
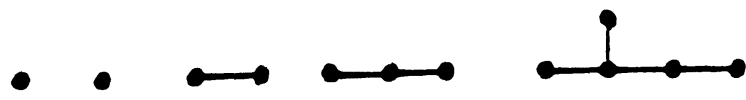

$4,8,52,56$

(6)
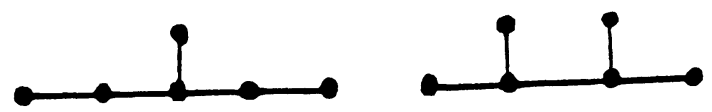

(7)
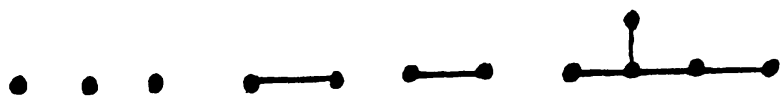

6,54

(8).
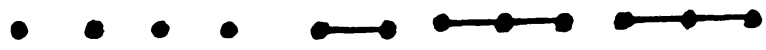

$10,18,24,34$

(9).

(10).

(11).
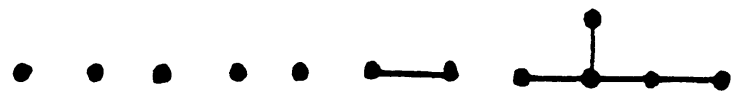

$11,17,42,46$

(12).

(13).

-
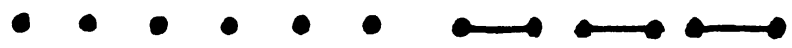

$13,15,40,48$

$12,16,41,47$

$9,19,25,33$
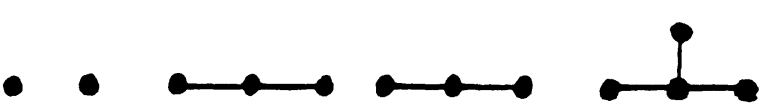

$14,39,43,45$

(14) .
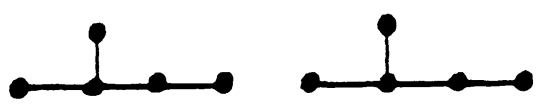

$20,26,32,49$

$21,27,31,50$ 
(15) .
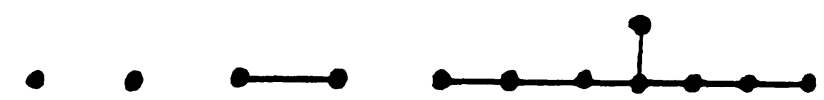

$28, \quad 30$

(16).
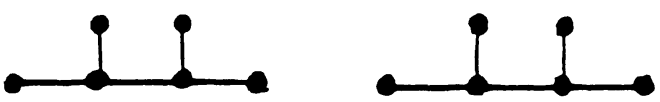

29,37

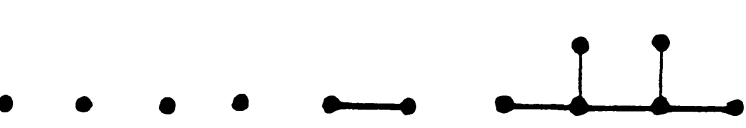

$(: 7)$

38,44

According to the above figure, the vertices of the graph of Biggs and Hoare (Figure 1 in [1]) can be roughly divided into 17 different sets (e.g., set (1) consists of vertices 1 and 36, etc.). Therefore, this graph has at least 17 different types of vertices and so it is much less symetrical than all known cages. The author could not get a reasonably good design of this graph. This, together with the graph given above seems to suggest that $f(3,9) \neq 58$.

For the other known cages (except those obtained by using the properties of finite projective geometry), a partial solution which contains about $80 \%$ of the total number of edges of a cage can always be completed to yield a cage of that type. For example, in order to shorten the computer program (used in [2]) to obtain a $(7,6)$-cage, about 758 of the total number of edges of this cage was completed by a computer. Only a few partial solutions were obtained, each of which was checked by hand to determine whether it is a solution. The author assumed that this was true for all other cages. However, the graph given here disproves this conjecture. This also seems to confirm that the construction of a $(3,9)$-cage is indeed a very difficult problem.

\section{REFERENCES}

1. Biggs, N. L. and Hoare, M. J. A trivalent graph with 58 vertices and girth 9 , Discrete Math. 20 (1980) 299-301.

2. O'Keefe, M. and Wong, P. K. The smallest graph of girth 6 and valency 7, J. Graph Theory $\underline{5}$ (1981) 79-85.

3. Wong, P. K. Cages - A survey, J. Graph Theory 6 (1982) 1-22.

4. Wong, P. K. On the smallest graphs of girth 10 and valency 3 , Discrete Math. 43 (1983) 199-124. 


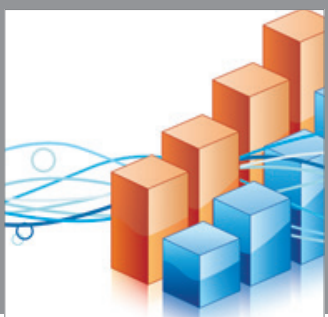

Advances in

Operations Research

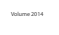

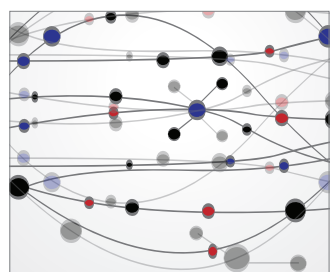

\section{The Scientific} World Journal
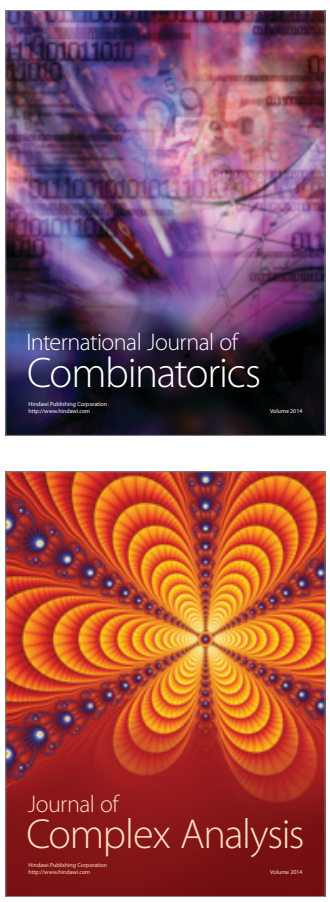

International Journal of

Mathematics and

Mathematical

Sciences
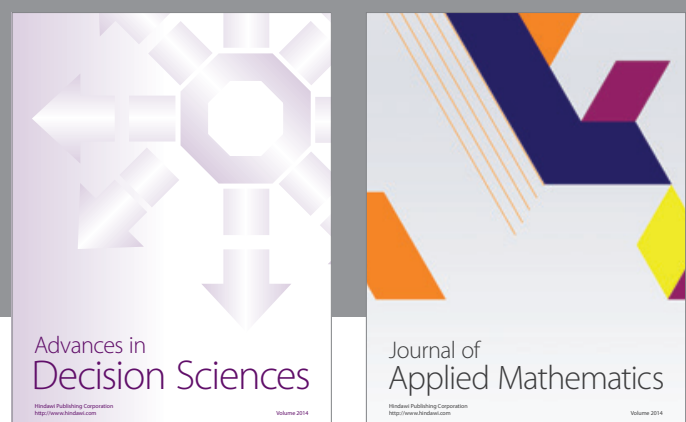

Journal of

Applied Mathematics
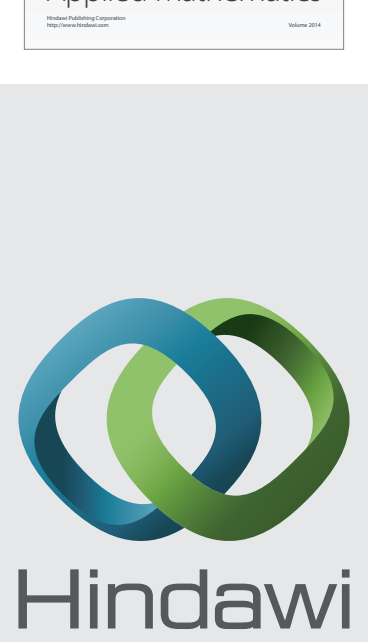

Submit your manuscripts at http://www.hindawi.com
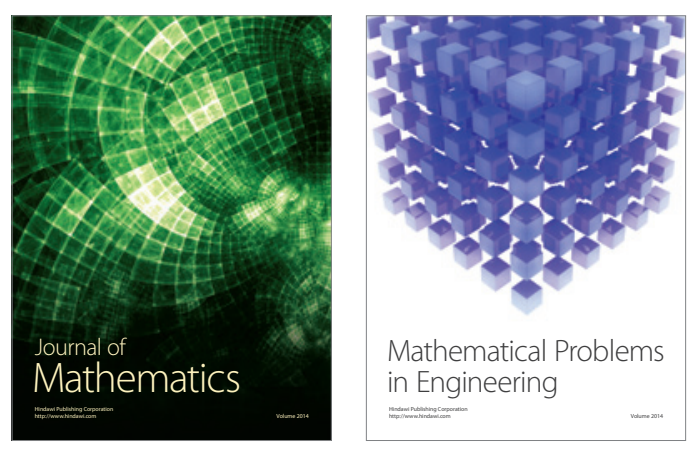

Mathematical Problems in Engineering
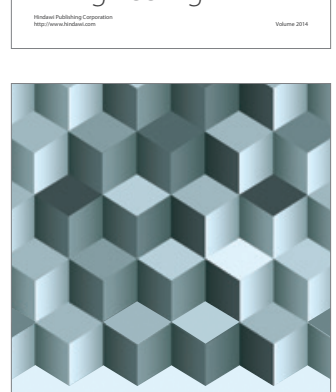

Journal of

Function Spaces
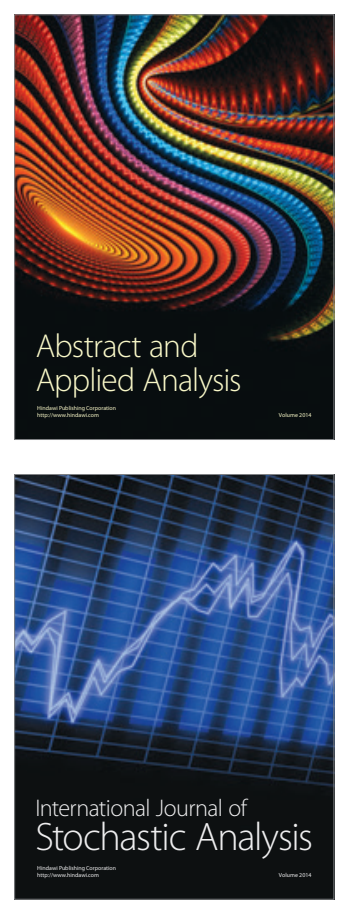

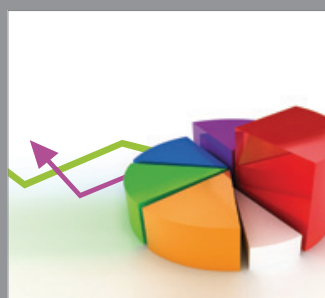

ournal of

Probability and Statistics

Promensencen
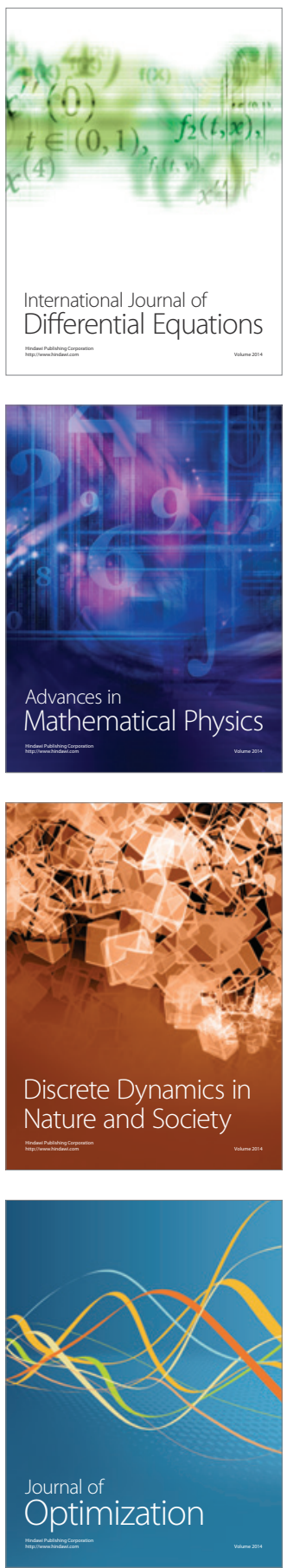